\title{
Specific and Rapid Reverse Assaying Protocol for Detection and Antimicrobial Susceptibility Testing of Pseudomonas Aeruginosa based on Bacteriophage Tail Fiber Protein and Magainin II Recognition
}

\section{Yong He ( $\nabla$ heyongzmu@163.com )}

Affiliated Hospital of Zunyi Medical College

\section{Hang Zhao}

Affiliated Hospital of Zunyi Medical College

Yuanwen Liu

Affiliated Hospital of Zunyi Medical College

He Zhou

Zunyi institute for food and drug control

\section{Research Article}

Keywords: Antimicrobial resistance, Antimicrobial susceptibility testing, Bacteriophage tail fiber protein, Magainin II, Pseudomonas aeruginosa

Posted Date: December 28th, 2020

DOI: https://doi.org/10.21203/rs.3.rs-130554/v1

License: (c) (i) This work is licensed under a Creative Commons Attribution 4.0 International License. Read Full License 


\section{Abstract}

The worldwide emergence and spread of antimicrobial resistance are accelerated by irrational administration and use of empiric antibiotic. A key point to the crisis is lack of rapid diagnostic protocol to antimicrobial susceptibility testing (AST) for timely and rational antibiotic prescription. Here, bacteriophage tail fiber protein (TFP) recombined in Escherichia coli expression system was functionalized on magnetic particles (MPs) to specifically capture $P$. aeruginosa, and FITC-labeledmagainin II was utilized as the indicator. For solving the MPs' blocking effects, a reverse assaying protocol (RAP) based on TFP recognition was investigated the feasibility of detection and AST of $P$. aeruginosa. $P$. aeruginosa detection can be rapidly, sensitively and specifically detected within $1.5 \mathrm{~h}$ with a linear range of $1.0 \times 10^{2}$ to $1.0 \times 10^{6} \mathrm{CFU} \cdot \mathrm{mL}^{-1}$ and a detection limit of $3.3 \times 10 \mathrm{CFU} \cdot \mathrm{mL}^{-1}$. Subsequently, the results of AST which was consistent in the results of broth dilution can be obtained within $3.5 \mathrm{~h}$. Due to the high specificity of TFP, the AST can actually be conducted without the requirement of bacterial isolation and identification by this RAP. Based on the proof-of-principle work, the detection and AST of other pathogens can be extended by expressing the TFP of their bacteriophages.

\section{Introduction}

Antimicrobial resistance (AMR) is a worldwide health crisis resulting in growing economic burden and increasing mortality ${ }^{1,2}$. The most important strategies to minimize AMR are the development of rapid and accurate diagnostic protocols for antimicrobial susceptibility testing (AST), which facilitating the timing of prescription to use the effective antibiotics ${ }^{3,4}$. Therefore, numerous efforts are exerted on developing rapid, sensitive and acute detection and AST for bacterial infection.

Traditional bacterial growth-based protocols are considered as the gold standard methods for bacterial detection and AST. These protocols show ideal repeatability, high standardization and good reliability. However, the isolation and identification procedure of bacterial detection usually require tedious process of 24-48 $\mathrm{h}^{5}$. The subsequent broth dilution or disk diffusion for AST performance require another bacterial growth cultured with given concentrations of various antibiotics, which demands almost another 24-48 $h^{6,7}$. Lack of timely and accurate results of bacterial AST results in frequent empiric antibiotic therapy, causing irrational antibiotic use and the development of $\mathrm{AMR}^{8}$. Therefore, some other bacterial growth-based protocols are reported to aim at shortening the time of AST, such as microscopy detection $^{9-11}$, electrochemical sensor ${ }^{12-14}$, phase-shift spectroscopy detection ${ }^{15}$, fluorescent detection ${ }^{16}$ and microfluidic devices based on slipchip technique ${ }^{17}$, surface-enhanced raman scattering ${ }^{18}$. Nevertheless, since lack of capacity of isolating the given bacterial species, they also require timeconsuming pretreatment procedure for bacterial culture, isolation and identification. Polymerase chain reaction-based protocols gradually attract attention to bacterial detection and performance of AST due to the advantages of rapidity, sensitivity and culture-free process ${ }^{20-22}$. However, they suffer from welltrained personnel, complicated molecular manipulation, prerequisite precise resistant gene information and frequent gene mutation. 
With the increasing global AMR, bacteriophages as the natural enemies to the bacteria have regained interest in solving the crisis caused by multi-resistant bacteria. Each strain of bacteria has one or more corresponding bacteriophages. Bacteriophages can highly specifically recognize their target bacteria even in harsh environment. Typical lifecycle of virulent bacteriophage involves the following steps: specific absorption on the bacterial cell wall, DNA injection into the bacterial cell, progenies replication and the lysis of bacteria cell for releasing progenies. Bacteriophages functional proteins (BFP) such as tail fiber protein (TFP), tailspike protein (TSP) and endolysin are the essential recognition elements which are responsible for absorption, injection and lysis, respectively ${ }^{23}$. Therefore, BFPs assumes the ideal molecular recognition attributes of high specificity, robustness, good anti-interference capability and universality to each bacterium ${ }^{23}$. Unfortunately, just like the bacteriophage entity, TSP and endolysin both have the inherent lytic activity which could be unfavorable for bacteria capture and sample manipulation.

In the previous work, we had utilized to the Escherichia coli (E. coli) expression system to produce the TFP of $P$ seudomonas aeruginosa ( $P$. aeruginosa) ${ }^{24}$. This recombinant TFP can specifically recognize $P$. aeruginosa without lytic activity. To investigate the application of TFP in AST detection, TFPfunctionalized magnetic particles were utilized to specifically capture $P$. aeruginosa, and fluorescein isothiocyanate (FITC) labeled magainin II was utilized as the fluorescent tracer. A reverse assaying protocol (RAP) combined magnetic separation was developed to specific, rapid and sensitive detection and AST of $P$. aeruginosa.

\section{Experimental}

\subsection{Instrumentations}

Scanning electron micrograph (SEM) was recorded by an S-3000N scanning electron microscope (Hitachi, Japan). Fluorescence (FL) signals were obtained from an Infinite M200 PRO microplate reader (TECAN, Switzerland). FL micrographs were recorded by using a NI - U FL microscope (Nikon, Japan).

\subsection{Reagents and materials}

Piperacillin/Tazobactam (PIP/TAZ), ceftazidime (CAZ), tobramycin (TOB), gentamicin (GEN) and levofloxacin (LVX) were all purchased from Solarbio Life Sciences (China). Strains of $P$. aeruginosa, $E$. coli, Pseudomonas solanacearum (P. solanacearum), Salmonella typhimurium (S. tyhimurium), Staphylococcus aureus (S. aureus), Staphylococcus epidermidis (S. epidermidis), Streptococcus mutans (S. mutans) were purchased from Guangdong Microbiology Culture Center (China). FITC labeled magainin II were obtained from GL Biochem (Shanghai) Ltd.. (China). Tetraethyl rhodamine isothiocyanate (TRITC) and gelatin were provided by Sigma-Aldrich (USA, www.sigma-aldrich.com). AffiAmino magnetic particles (MPs) were purchased from Lab on a Bead AB (Sweden) in which activation buffer and blocking buffer were provided. Both human urine collected from the authors and rat plasma gifted by another lab were used as the common matrices. Luria - Bertani (LB) broth consisted of $10 \mathrm{~g} \cdot \mathrm{L}^{-1}$ 
$\mathrm{NaCl}, 5 \mathrm{~g} \cdot \mathrm{L}^{-1}$ yeast extract and $10 \mathrm{~g} \mathrm{~L}^{-1}$ tryptone. Washing buffer was composed of $10 \mathrm{mM}$ PBS $(\mathrm{pH} 7.4)$ and $0.5 \%$ tween-20. Human beings aren't involved in the study.

\subsection{Bacterial culture and counting}

Strains of $P$. aeruginosa and other bacteria were grown in $30 \mathrm{~mL}$ of LB broth with continuous shaking at $80 \mathrm{rpm}$ and $37^{\circ} \mathrm{C}$ under aerobic condition until the value of $\mathrm{OD}_{600}$ reached 1.0. For bacterial detection, $10 \mathrm{mM}$ PBS ( $\mathrm{pH}$ 7.4) was utilized to serially dilute the bacterial culture to reach the target concentration. The bacterial concentrations were evaluated according to standard approach of bacterial culture and counting.

\subsection{Preparation procedure of TRITC-labeled TFP}

One milliliter of TFP solution at the concentration of $1.0 \mathrm{mg} \cdot \mathrm{mL}^{-1}$ was slowly mixed with $1.0 \mathrm{~mL}$ of TRITC solution at $3.0 \mathrm{mg} \cdot \mathrm{mL}^{-1}$ dissolved in dimethyl sulfoxide, followed by 12 -h reaction at $4{ }^{\circ} \mathrm{C}$. Subsequently, $\mathrm{NH}_{4} \mathrm{Cl}$ solution was used to stop the reaction. Finally, the solution was dialyzed with $10 \mathrm{mM}$ PBS ( $\mathrm{pH} 7.4)$ for $48 \mathrm{~h}$ at room temperature.

\subsection{Fluorescent microscope image of stained $P$. aeruginosa}

One milliliter of $P$. aeruginosa suspension at $1.0 \subseteq 10^{6} \mathrm{CFU} \cdot \mathrm{mL}^{-1}$ was added with $200 \mu \mathrm{L}$ of FITC labeled magainin II at $20.0 \mu \mathrm{g} \cdot \mathrm{mL}^{-1}$ and the equal volume of TRITC labeled TFP. After 1-h incubation at room temperature, the suspension was centrifuged at $2500 \mathrm{~g}$ and washed thrice, followed by resuspended in $10 \mathrm{mM}$ PBS ( $\mathrm{pH}$ 7.4). Subsequently, $10 \mu \mathrm{L}$ of the stained $P$. aeruginosa suspension was observed by a FL microscope with the magnificence of 1000 . The excitation wavelength of TRITC and FITC were $544 \mathrm{~nm}$ and $488 \mathrm{~nm}$, respectively, and the emission wavelength of TRITC and FITC were $570 \mathrm{~nm}$ and $525 \mathrm{~nm}$, respectively.

\subsection{Preparation procedure of TFP-functionalized MPs}

TFP-functionalized MPs were obtained according to manufacturer guideline. Briefly, after washed by washing buffer, $100 \mu \mathrm{L}$ of MPs was resuspended into the $1.0 \mathrm{~mL}$ of washing buffer. Subsequently, $50 \mu \mathrm{L}$ of the activation buffer was mixed with the above suspension for 15 min activation. After the MPs were thoroughly washed, $1.0 \mathrm{~mL}$ of TFP solution at $100 \mu \mathrm{g} \cdot \mathrm{mL}^{-1}$ was added for 1 -h reaction at room temperature, followed by another thorough washing. Then the residue active sites of MPs were blocked with $80 \mu \mathrm{L}$ of the blocking buffer and $1 \%$ gelatin at room temperature for 45 min. Finally, after washed thrice the TFP-functionalized MPS were stored in $10 \mathrm{mM}$ PBS $(\mathrm{pH} 7.4)$ at $4{ }^{\circ} \mathrm{C}$.

\subsection{RAP for $P$. aeruginosa}

One milliliter of $P$. aeruginosa suspension was added with $10 \mu \mathrm{L}$ of TFP-functionalized MPs for a 45 min incubation at room temperature. After magnetic separation and thorough washing by the washing buffer thrice, the TFP-functionalized MPs was re-suspended into $100 \mu \mathrm{L}$ of $10 \mathrm{mM}$ PBS (pH 7.4). Subsequently, the suspension was mixed with $100 \mu \mathrm{L}$ of FITC labeled magainin II at $20.0 \mu \mathrm{g} \cdot \mathrm{mL}^{-1}$ for another $45 \mathrm{~min}$ 
incubation at room temperature. Finally, after the MPs complex was separated, the supernatant solution was moved into a 96-well microplate to obtain the FL signal with the excitation wavelength and emission wavelength of $488 \mathrm{~nm}$ and $525 \mathrm{~nm}$, respectively.

\subsection{AST of P. aeruginosa}

The stock solution of antibiotics were prepared according to Performance Standards for Antimicrobial Susceptibility Testing of Clinical and Laboratory Standards Institute (CLSI) M100S (29th Edition). Five hundred microliters of bacterial suspensions were mixed with the same volume of serial concentrations of antibiotics solution at $37^{\circ} \mathrm{C}$ for $2 \mathrm{~h}$. With the above RAP for $P$. aeruginosa detection, the susceptibility of $P$. aeruginosa was assessed by the change of bacterial concentrations which was measured by the $\mathrm{FL}$ signal.

\section{Results And Discussion}

\subsection{The principle of RAP for $P$. aeruginosa detection}

As shown in Fig. 1, we developed a RAP for $P$. aeruginosa detection. Nonlytic recombinant TFP was functionalized on the MPs to specifically capture $P$. aeruginosa through specific interaction between TFP and the lipopolysaccharide on the bacterial cell wall 24,25 . FITC labeled magainin II anchoring on the cytoplasmic membrane of both G-positive and G-negative bacteria was utilized as the fluorescent tracer $^{26}$. When $P$. aeruginosa was captured by the TFP-functionalized MPs to form bacteria-MPs complex, quantitative excess FITC labeled magainin II was added with these complex. After FITC-magainin IIbacteria-MPs complex was magnetically separated, the supernatant solution was transferred into the microplate to obtain the FL intensity. The changed values of FL intensity $(\Delta \mathrm{FL})$ was utilized to quantitate $P$. aeruginosa, where $\Delta \mathrm{FL}$ was defined as the following equation: $\Delta \mathrm{FL}=\mathrm{FL}_{\text {blank sample }}-\mathrm{FL}_{\text {sample}}$. Compared to direct assaying protocol, RAP can solve the blocking effects of the MPs to the intensity of FITC since the size of MPs was much bigger than that of FITC. When the FITC-magainin II-bacteria-MPs complex was formed, only a few fraction of fluorescent tracer which was on the side of exciting light of the complex can be triggered to emit the FL signals.

To testify the coinstantaneous binding capability of TFP and magainin II to $P$. aeruginosa, TRITC labeled TFP and FITC labeled magainin II were simultaneously mixed with $P$. aeruginosa to stain the cells of $P$. aeruginosa. As shown in Fig. 2, red FL from TRITC and green FL from FITC can be both observed on the surface of $P$. aeruginosa cells. This phenomenon demonstrated that TFP and magainin II can simultaneously bind with $P$. aeruginosa at different sites to form the sandwich complex.

\subsection{Characterization of capture of by TFP-functionalized MPs}

To investigate the capture capacity of TFP-functionalized MPs to $P$. aeruginosa, SEM was utilized to observe the capture behavior of TFP-functionalized MPs. As shown in Fig. 3, compared to the bare MPs, 
$P$. aeruginosa cells were bound and observed on the surface of TFP-functionalized MPs. This demonstrated that after functionalized on MPs TFP remained the binding capacity to $P$. aeruginosa.

\subsection{Condition optimization of P. aeruginosa detection}

To enhance the sensitivity of RAP for $P$. aeruginosa detection, the following parameters were evaluated including (1) the amount of TFP-functionalized MPs, (2) the incubation time for P. aeruginosa and FITC labeled magainin II, (3) the concentration of FITC labeled magainin II. As illustrated in Figure S1-S3, the values of $\triangle F L$ reached optimal when the chosen parameters were as follows: (1) $10 \mu \mathrm{L}$ of TFPfunctionalized MPs; (2) 45 min incubation time for $P$. aeruginosa; (3) 45 min incubation time for FITC labeled magainin II; and (4) $20.0 \mu \mathrm{g} \cdot \mathrm{mL}^{-1}$ of FITC labeled magainin II.

\subsection{Detection performance}

Under the optimal experimental conditions, RAP for $P$. aeruginosa detection showed a linear range of 1.0 $\times 10^{2}$ to $1.0 \times 10^{6} \mathrm{CFU} \cdot \mathrm{mL}^{-1}$ with the detection limit of $3.3 \times 10 \mathrm{CFU} \cdot \mathrm{mL}^{-1}$. The regression equation was $\lg \Delta F L$ (a.u.) $=1.41+0.404 \mathrm{lg} C\left(\mathrm{CFU} \cdot \mathrm{mL}^{-1}\right)$ with a correlation coefficient of 0.9952 (Figure S4). Here $\Delta F L$ and $C$ represent the changed values of FL intensity and the concentration of $P$. aeruginosa. The relative standard deviation (RSD) values at low $\left(1.0 \times 10^{2} \mathrm{CFU} \cdot \mathrm{mL}^{-1}\right)$, medium $\left(1.0 \times 10^{4} \mathrm{CFU} \cdot \mathrm{mL}^{-1}\right)$ and high $\left(1.0 \times 10^{6} \mathrm{CFU} \cdot \mathrm{mL}^{-1}\right)$ concentrations were $6.44 \%, 3.67 \%$ and $2.18 \%$, respectively. This results demonstrated RAP showed acceptable repeatability.

\subsection{Specificity}

The specificity of RAP was investigated by selecting three Gram-negative bacteria ( $E$. coli, $P$. solanacearum and $S$. tyhimurium) and three Gram-positive bacteria (S. aureus, S. epidermidis and $S$. mutans). The concentrations of these interference bacteria were all $1.0 \times 10^{5} \mathrm{CFU} \cdot \mathrm{mL}^{-1}$ for the specificity investigation. The specificity of RAP was calculated by the designed interference degree (ID) values of the above interference bacteria in the following equation.

$\mathrm{ID}=\Delta F L_{\text {interference bacteria }} / \Delta F L_{P . \text { aeruginosa }} \subseteq 100 \%(1)$

As illustrated in Fig. 4, the ID values of the tested interference bacteria were all below 5.26\%. For the further investigation of potential interference to $P$. aeruginosa detection, Mixture A was composed of all the six interference bacteria and Mixture B was prepared by mixing $P$. aeruginosa with Mixture A. The ID value of Mixture A was $4.37 \%$. Compared to that of $P$. aeruginosa, the $\Delta \mathrm{FL}$ intensity of Mixture B showed the minor difference (3.14\%). Therefore, RAP for $P$. aeruginosa detection showed good specificity.

\subsection{Practical sample detection}

To investigate the potential application of this RAP for $P$. aeruginosa detection, $5 \%$ glucose injection, rat plasma and human urine were spiked with $P$. aeruginosa suspension at given concentrations. As shown in Table 1, the recovery values ranged from $90.1-104.2 \%$, with the RSD all below $5.0 \%$. This results demonstrated the reliability of RAP for detecting $P$. aeruginosa in complicated matrix. 
Table 1

Recovery tests for $P$. aeruginosa detection spiked in practical samples $(n=4)$.

\begin{tabular}{|c|c|c|c|}
\hline Sample & Spiked (CFU mL ${ }^{-1}$ ) & Recovery (\%) & RSD (\%) \\
\hline \multirow[t]{4}{*}{ Glucose injection } & $1.0 \times 10^{6}$ & 104.2 & 3.2 \\
\hline & $1.0 \times 10^{5}$ & 95.6 & 3.1 \\
\hline & $1.0 \times 10^{4}$ & 98.7 & 2.8 \\
\hline & $1.0 \times 10^{3}$ & 93.4 & 4.6 \\
\hline \multirow[t]{4}{*}{ Human urine } & $1.0 \times 10^{6}$ & 97.6 & 4.5 \\
\hline & $1.0 \times 10^{5}$ & 98.9 & 4.7 \\
\hline & $1.0 \times 10^{4}$ & 93.9 & 2.7 \\
\hline & $1.0 \times 10^{3}$ & 92.2 & 5.0 \\
\hline \multirow[t]{4}{*}{ Rat plasma } & $1.0 \times 10^{6}$ & 101.6 & 2.1 \\
\hline & $1.0 \times 10^{5}$ & 93.5 & 3.8 \\
\hline & $1.0 \times 10^{4}$ & 97.2 & 4.6 \\
\hline & $1.0 \times 10^{3}$ & 90.1 & 3.9 \\
\hline
\end{tabular}

\subsection{AST of P. aeruginosa}

AST of $P$. aeruginosa was evaluated by detecting the $\Delta \mathrm{FL}$ signals of $1.0 \subseteq 10^{5} \mathrm{CFU} \cdot \mathrm{mL}^{-1} P$. aeruginosa cultured with serial concentrations of antibiotics. According to the guidance of CLSI M100S, the four antibiotics of group A including PIP/TAZ, CAZ, TOB and GEN and one antibiotic of group B selected as LVX were utilized to validate the AST of $P$. aeruginosa to demonstrate its reliability. After $P$. aeruginosa was cultured with the absence (blank group, BG) and the presence (test group, TGs) of serial concentrations of antibiotics for $2 \mathrm{~h}$ at $37^{\circ} \mathrm{C}$, the $\Delta \mathrm{FL}$ signals of $P$. aeruginosa were calculated and compared. The same amount of $P$. aeruginosa suspension stored at $4{ }^{\circ} \mathrm{C}$ was detected as the control group (CPs). Since $P$. aeruginosa at $4{ }^{\circ} \mathrm{C}$ grew extremely slowly, the concentrations of $P$. aeruginosa was considered as remaining almost unchanged. The results of AST were obtained through comparing the $\triangle F L$ signals of TGs with those of CGs and TGs.

For the AST of $P$. aeruginosa to PIP/TAZ, at the concentration range from $16 / 4$ to $128 / 4 \mu \mathrm{g} \cdot \mathrm{mL}^{-1}$, the $\triangle \mathrm{FL}$ signals of TGs were about $99.7 \%$ and $29.7 \%$ of those of $C G$ s and $B G s$, respectively (Fig. $5 \mathrm{~A}$ ). As 
shown in Fig. 5B-5D, the similar results were also found for CAZ $\left(8-32 \mu \mathrm{g} \cdot \mathrm{mL}^{-1}\right)$, TOB $\left(4-16 \mu \mathrm{g} \cdot \mathrm{mL}^{-1}\right)$ and GEN (4-16 $\left.\mu \mathrm{g} \cdot \mathrm{mL}^{-1}\right)$. This results demonstrated under the effect of these antibiotics concentration the growth of $P$. aeruginosa was significantly inhibited. The minimum inhibitory concentrations (MICs) of PIP/TAZ, CAZ, TOB and GEN were $<16 / 4,<8,<4$ and $<4 \mu \mathrm{g} \cdot \mathrm{mL}^{-1}$, respectively. According to the guidance of CLSI M100S (Table S1), P. aeruginosa was susceptible to these four antibiotics (Table 2). For the AST of $P$. aeruginosa to LVX, when the concentrations were 1 and $2 \mu \mathrm{g} \cdot \mathrm{mL}^{-1}$, the $\Delta \mathrm{FL}$ signals of TGs were about $312 \%$ and $93.2 \%$ of those of CGs and BGs, respectively (Fig. $5 \mathrm{E}$ ). This results demonstrated that the growth of $P$. aeruginosa was slightly inhibited by LVX in comparison with the normal growth of $P$. aeruginosa (BGs). However, the concentration of $\mathrm{LVX}$ reached $4 \mu \mathrm{g} \cdot \mathrm{mL}^{-1}$, the $\Delta \mathrm{FL}$ signals of TGs reduced to about $99.7 \%$ and $29.7 \%$ of those of CGs and BGs, respectively. It shown that the growth of $P$. aeruginosa was significantly influenced by LVX at the concentration of $4 \mu \mathrm{g} \cdot \mathrm{mL}^{-1}$. Therefore, the MIC of LVX was $4 \mu \mathrm{g} \cdot \mathrm{mL}^{-1}$ and $P$. aeruginosa was resistant to LVX (Table 2).

Table 2

The AST results of this protocol and CLSI data for $P$. aeruginosa (ATCC 27853).

$\mathrm{S}$ : susceptible, R: resistant, I: intermediate

\begin{tabular}{|lllllll|}
\hline Antibiotics & & PIP/TAZ & CAZ & TOB & GEN & LVX \\
\hline \multirow{2}{*}{ Testing results } & MIC $\left(\mu \mathrm{g} \mathrm{mL}^{-1}\right)$ & $<16 / 4$ & $<8$ & $<4$ & $<4$ & 4 \\
\cline { 2 - 7 } & Susceptibility & S & S & S & S & R \\
\hline \multirow{2}{*}{ CLSI data } & Susceptibility & S & S & S & S & I or R \\
\hline
\end{tabular}

The AST results for all the testing antibiotics were consistent with the provided data of the document of CLSI 100S. This results demonstrated that the RAP protocol showed good reliability for the AST.

\section{Conclusion}

In conclusion, a rapid, sensitive and specific RAP using TFP and magainin II as dual recognition elements was developed to perform the detection and AST of $P$. aeruginosa. Since TFP can specifically recognize the target cells of $P$. aeruginosa from other interference bacteria, the results of AST can actually be obtained within $4 \mathrm{~h}$ without the time-consuming process of bacterial isolation and identification, which can facilitate the decreasing frequency of irrational empiric antibiotic therapy. Based on this proof-ofprinciple work, the detection and AST of other bacteria can be facilely completed by the expression of the TFP of their bacteriophages. In the future work, we will focus on further reduce the detection time of AST based on TFP recognition through other detection technique such as microfluidic system or single-cell imaging.

\section{Declarations}

\section{Acknowledgements}


This work was financially supported by National Natural Science Foundation of China (21964023), Science and Technology Foundation of Guizhou Province (20201Y044) and Doctoral Initiation Fund of Zunyi Medical University (No. 5).

\section{Author contributions}

Conceived and designed the experiments: Y.H., H.Z., Y.W.L. and H.Z. Performed the experiments: Y.H., H.Z., Y.W.L. and H.Z. Wrote the paper: Y.H. and H.Z.

\section{Conflicts of interest}

The authors declare no conflicts of interest.

\section{References}

1. Laxminarayan, R. et al. Antibiotic resistance-the need for global solutions. Lancet Infect. Dis. 13, 1057-1098 (2013).

2. Woolhouse, M. \& Farrar J. An intergovernmental panel on antimicrobial resistance. Nature $509,555-$ 557 (2014).

3. Lee, J. H., Park, K. S., Karim, A. M., Lee, C. R. \& Lee, S. H. How to minimize antibiotic resistance. Lancet 16, 406-407 (2016).

4. Tillotson, G. Antimicrobial resistance: what's needed. Lancet Infect. Dis. 15, 758-760 (2015).

5. Behera, B. et al. Emerging technologies for antibiotic susceptibility testing. Biosens. Bioeletron. 142, 111552 (2019).

6. Jorgensen, J. H. \& Ferraro, M. J. Antimicrobial susceptibility testing: a review of general principles and contemporary practices. Clin. Infect. Dis. 49, 1749-1755 (2009).

7. Pulido, M. R., Garcia-Quintanilla, M., Martin-Pena, R., Cisneros, J. M. \& McConnell, M. J. J. Progress on the development of rapid methods for antimicrobial susceptibility testing. Antimicrob. Chemother. $682710-2717$ (2013).

8. Holmes, A. H. et al. Understanding the mechanisms and drivers of antimicrobial resistance. Lancet 387, 176-187 (2016).

9. Mo, M. N. et al. Rapid antimicrobial susceptibility testing of patient urine samples using large volume free-solution light scattering microscopy. Anal. Chem. 91, 10164-10171 (2019).

10. Choi, J. et al. Direct, rapid antimicrobial susceptibility test from positive blood cultures based on microscopic imaging analysis. Sci. Rep. 7, 1148 (2017).

11. Baltekin, O., Boucharin, A., Tano, E., Andersson, D. I. \& Elf, J. Antibiotic susceptibility testing in less than 30 min using direct single-cell imaging. Proc. Natl. Acad. Sci. U. S. A. 114, 9170-9175 (2017). 
12. Maiga, M. et al. A novel support for enzyme immobilization in bio-based electrochemical detection and its in vitro antimicrobial activity. Sens. Actuators B 235, 46-55 (2016).

13. Lee, K. S. et al. Electrical antimicrobial susceptibility testing based on aptamer-functionalized capacitance sensor array for clinical isolates. Sci. Rep. 10, 13709 (2020).

14. Zhang, X. Z. et al. Online monitoring of bacterial growth with an electrical sensor. Anal. Chem. 90, 6006-6011 (2018).

15. Leonard, H., Halachmi, S., Ben-Dov, N., Nativ, O. \& Segal, E. Unraveling antimicrobial susceptibility of bacterial networks on micropillar architectures using intrinsic phase-shift spectroscopy. ACS Nano 11, 6167-6177 (2017).

16. Greorchuk, B. S. J. et al. Antiseptic quaternary ammonium compound tolerance by gram-negative bacteria can be rapidly detected using an impermeant fluorescent dye-based assay. Sci. Rep. 10, 20543 (2020).

17. Yi, Q. L. et al. Direct antimicrobial susceptibility testing of bloodstream infection on SlipChip. Biosens. Bioeletron. 135, 200-207 (2019).

18. Chang, K. W., Cheng, H. W., Shiue, J., Wang, J. K., Wang, Y. L. \& Huang, N. T. Antibiotic susceptibility test with surface-enhanced raman scattering in a microfluidic system. Anal. Chem. 91, 10988-10995 (2019).

19. Li, H. et al. Adaptable microfluidic system for single-cell pathogen classification and antimicrobial susceptibility testing. Proc. Natl. Acad. Sci. U. S. A. 116, 10270-10279 (2019).

20. Athamanolap, P., Hsieh, K., O’Keefe, C. M., Zhang, Y., Yang, S. \& Wang, T. H. Nanoarray digital polymerase chain reaction with high-resolution melt for enabling broad bacteria identification and pheno-molecular antimicrobial susceptibility test. Anal. Chem. 91, 12784-12792 (2019).

21. Schoepp, N. G. et al. Digital quantification of DNA replication and chromosome segregation enables determination of antimicrobial susceptibility after only 15 minutes of antibiotic exposure. Angew. Chem. Int. Ed. 128, 9709-9713 (2016).

22. Boehme, C. C. et al. Rapid molecular detection of tuberculosis and rifampin resistance. New Engl. J. Med. 363, 1005-1015 (2010).

23. Kutter, E. \& Sulakvelidze, A. Phages: Biology and Applications (CRC Press, 2005).

24. He, Y. et al. Nonlytic recombinant phage tail fiber protein for specific recognition of Pseudomonas aeruginosa. Anal. Chem. 90, 14462-14468 (2018).

25. Garcia-Doval, C. \& van Raaij, M. J. Structure of the receptor-binding carboxy-terminal domain of bacteriophage T7 tail fibers. Proc. Natl. Acad. Sci. U. S. A. 109, 9390-9395 (2012).

26. Fjell, C. D., Hiss, J. A., Hancock, R. E. W. \& Schneider, G. Designing antimicrobial peptides: form follows function. Nat. Rev. Drug. Discov. 11, $37-51$ (2012).

\section{Figures}




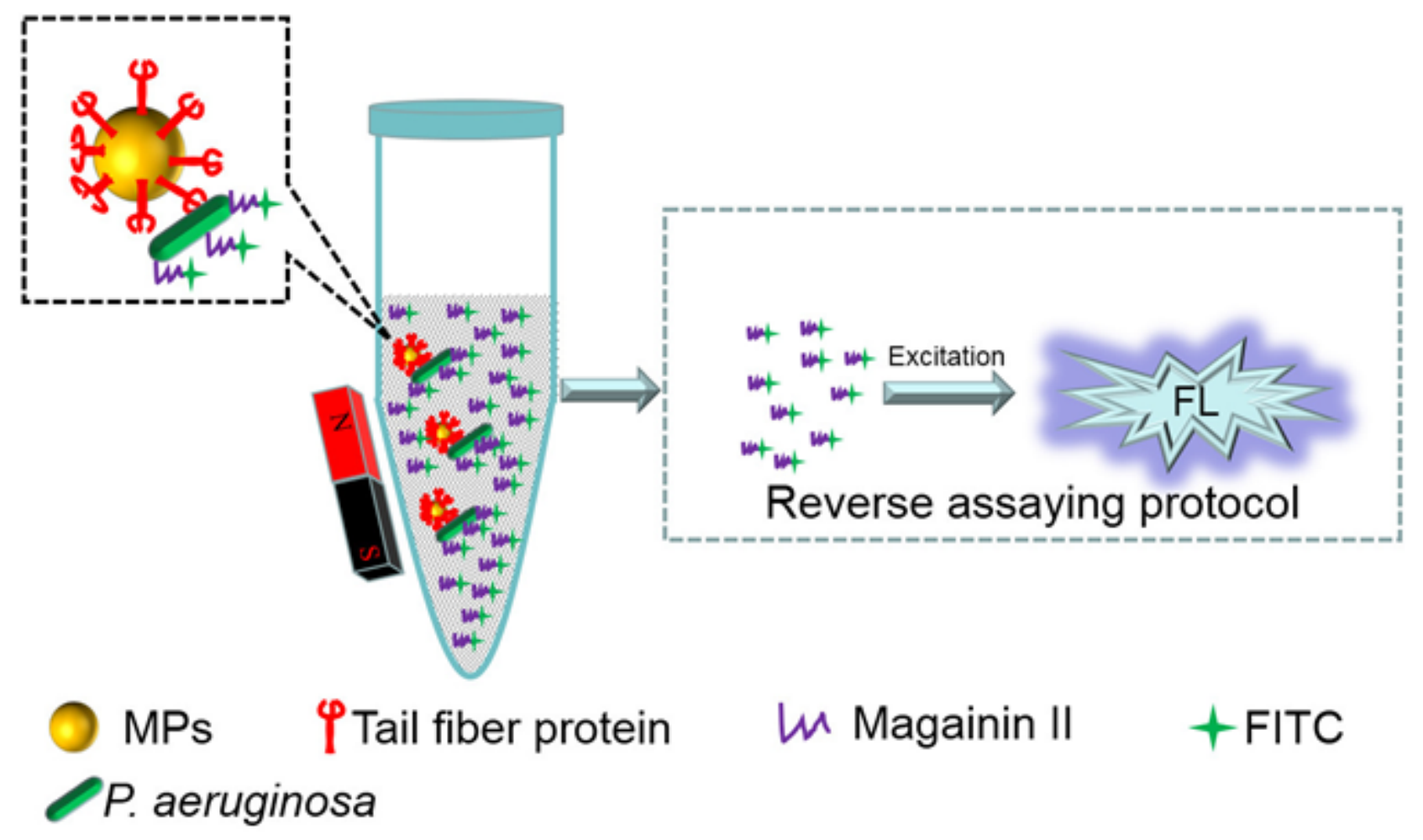

Figure 1

Schematic illustration of RAP for P. aeruginosa detection.

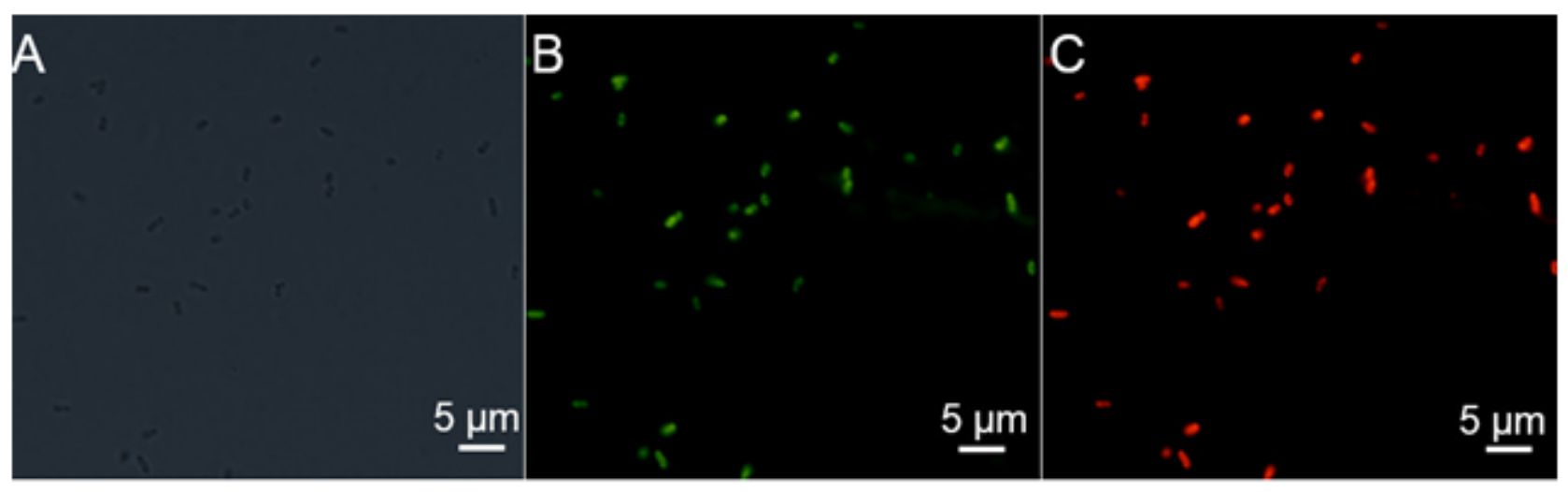

Figure 2

FL microscope image of the stained P. aeruginosa. (A) bright field, (B) green FL channel, (c) red FL channel. 

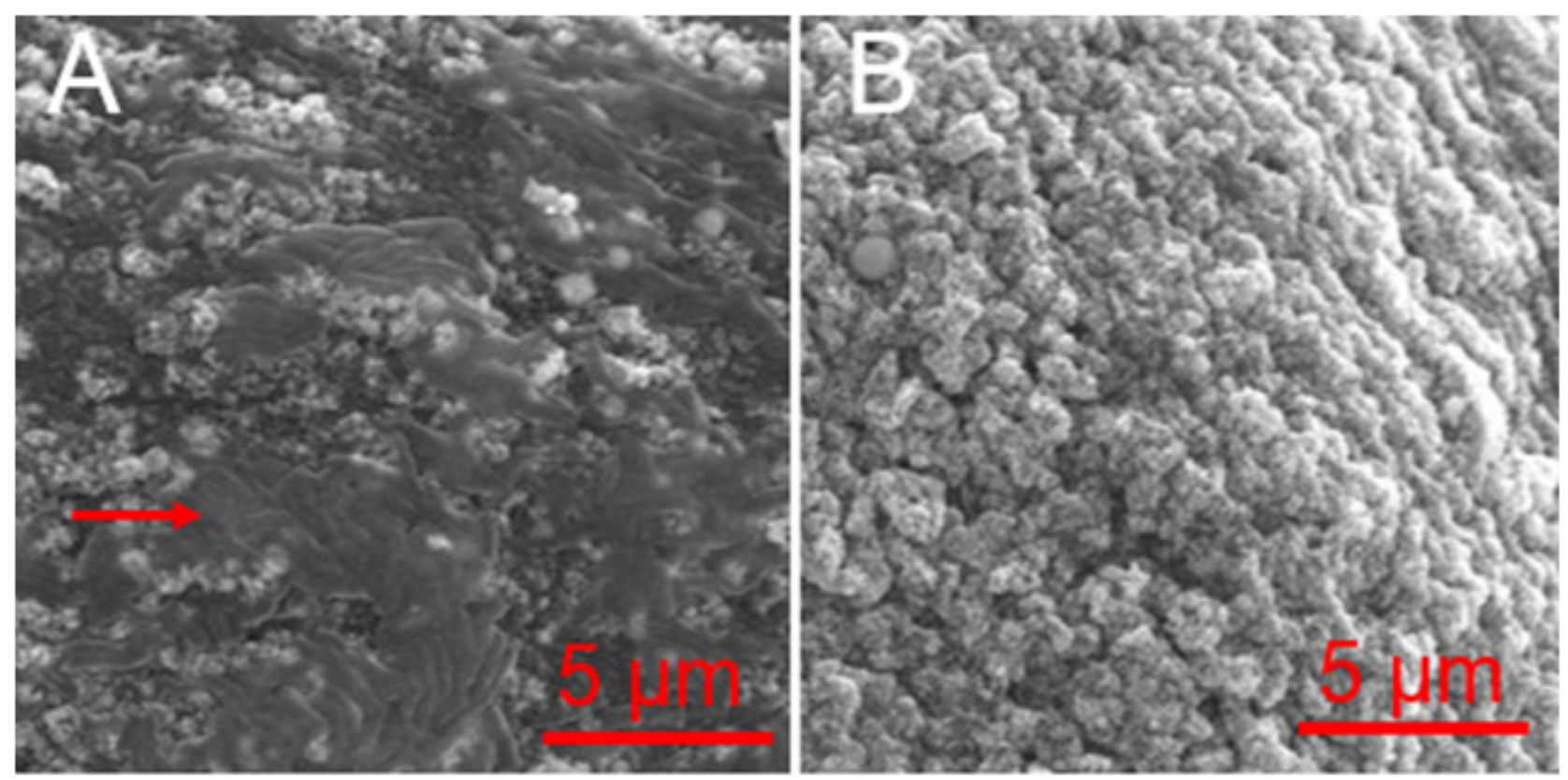

\section{Figure 3}

SEM image of (A) P. aeruginosa captured on the surface of TFP-functionalized MPs and (B) bare surface of TFP-functionalized MPs. The red arrow shows the captured P. aeruginosa. 


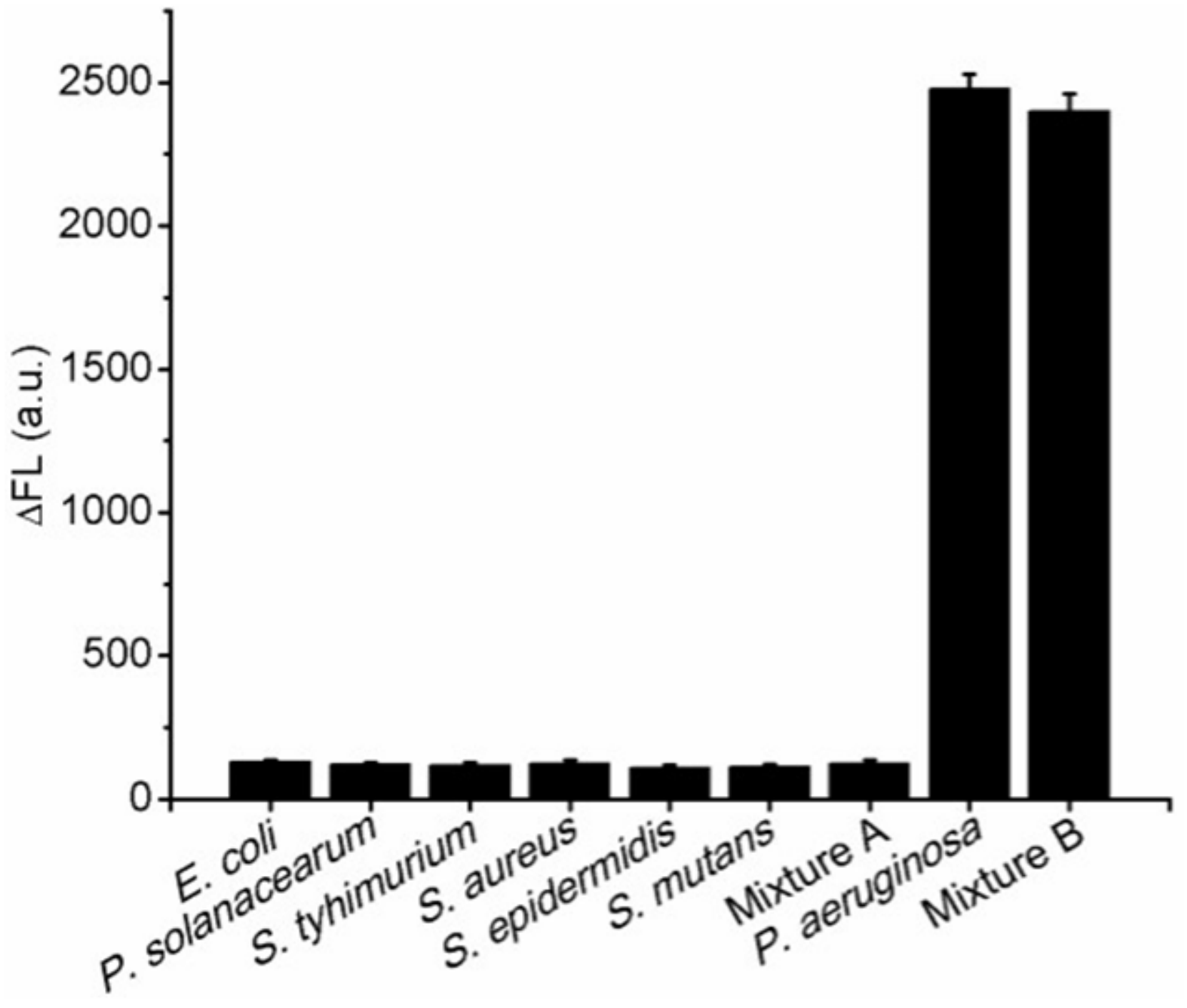

Figure 4

Specificity of RAP for P. aeruginosa detection. The concentrations of all the tested bacteria were $1.0 \times 105$ $\mathrm{CFU} \cdot \mathrm{mL}-1(n=4)$. 

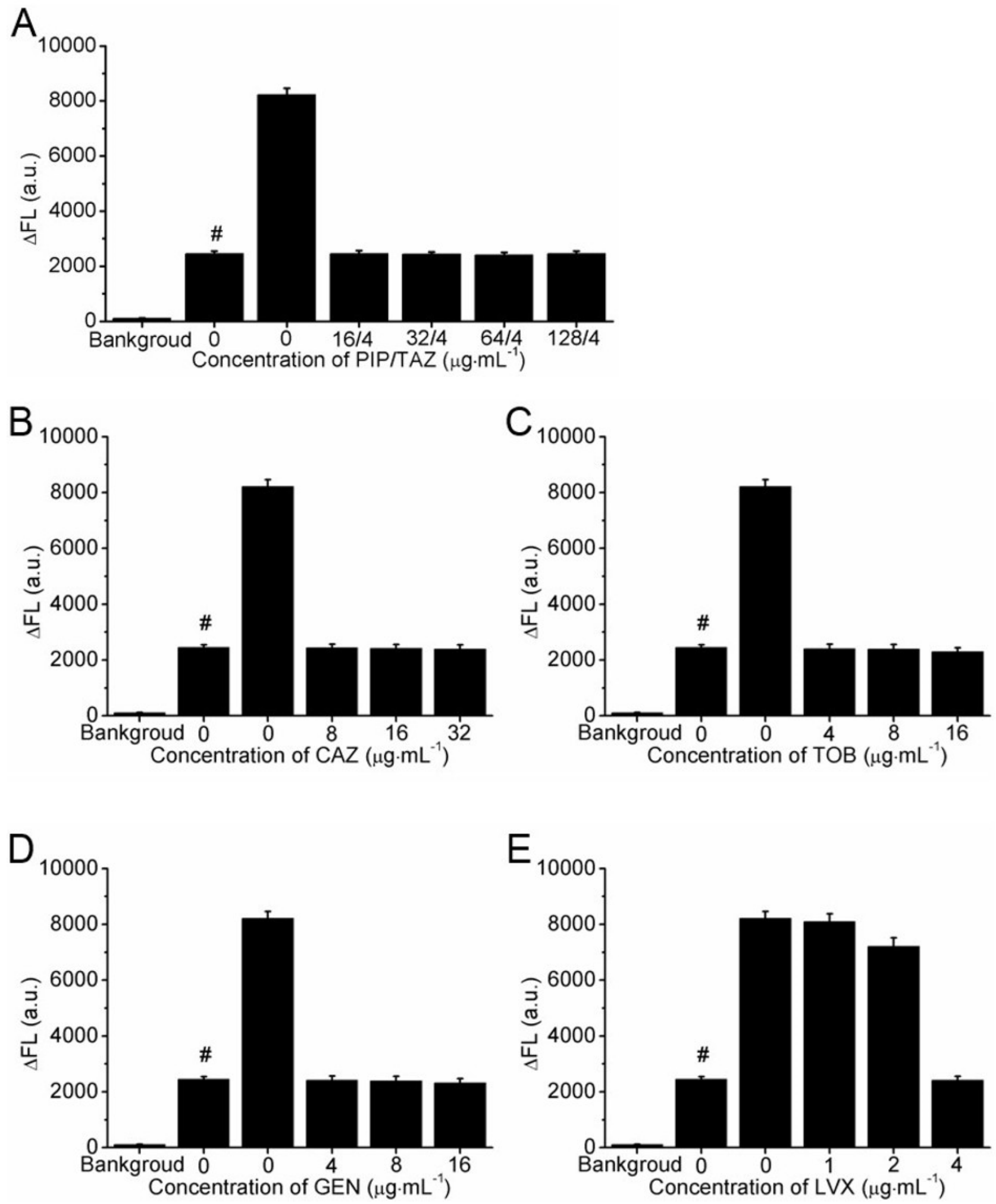

Figure 5

AST of P. aeruginosa (ATCC 27853) treated by (A) PIP/TAZ, (B) CAZ, (C) TOB, (D) GEN and (E) LVX. Background signals represent the $\triangle F L$ from PBS. \# signifies the $P$. aeruginosa suspension was kept at 4 ${ }^{\circ} \mathrm{C}$ before the performance of AST $(n=4)$.

\section{Supplementary Files}


This is a list of supplementary files associated with this preprint. Click to download.

- Supportinginformation.doc 